\section{A METHOD OF TAKING IMPRESSIONS OF THE WEIGHT-BEARING SURFACE OF THE FOOT.}

BY W. DUNCAN LAWRIE, M.D. EDIN., F.R C.S. EDIN., HOYORARY SURGEON TO THE IBIRMINGHAM CRIPPLE CHILDREN'S UNION.

ThE method usually recommended of obtaining impressions of the foot-viz., by standing on a smoked paper-has the disadvantage that for a permanent record the impression requires to be varnished. A method recently advocated by Schüman of Leipsic ${ }^{1}$ gets over the difficulty by employing two solutions--one a solution of ferric chloride (half strength

FIC. 1.

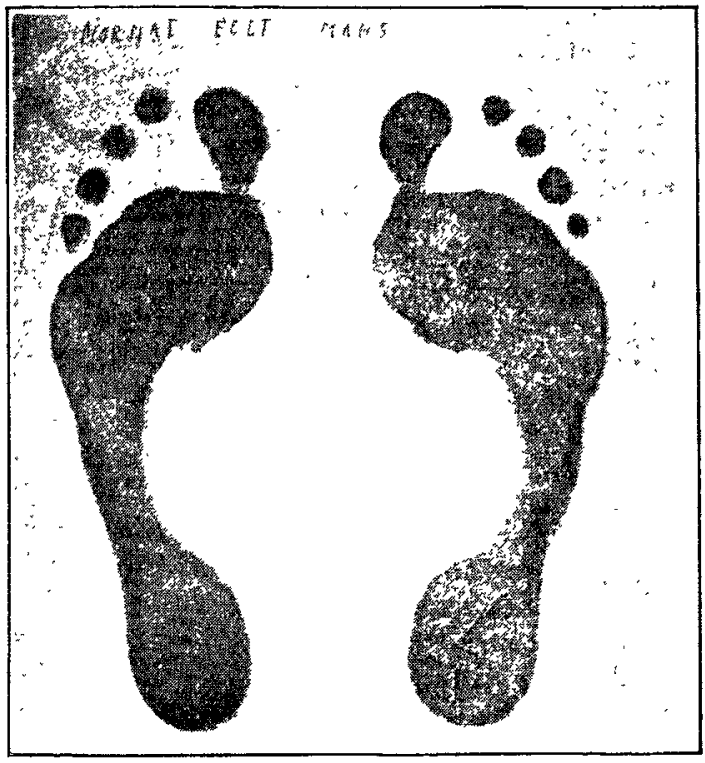

The impression of normal feet.

B.P. liq.), with which the paper is thinly coated and after wards dried, and the other a 5 per cent. solution of ferrocyanide of potash. The foot is painted with the ferrocyanide solution and then placed on the paper and a blue impression is left which is permanent and requires no aftertreatment. The preparation of the paper, however, is

FIG. 2.

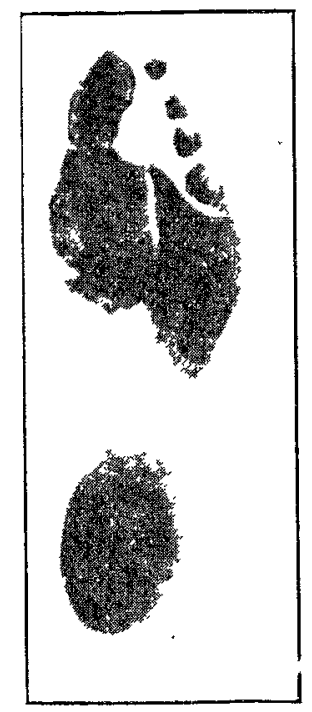

Showing the result of high-heeled boots.

troublesome and it is difficult to get the foot evenly wetted with the ferro-cyanide solution.

The method advocated in this paper is that employed in taking impressions of finger-tips for identification purposes. The materials required are a glass slab, say 16 inches by

Münchener Medicinische Wochenschrift, Jan. 6th, 1906.
13 inches, a roller, and a tube of specially prepared printer's ink. A thin film is pressed out on the glass plate by the roller, and after standing on this the patient transfers his blackened sole to a sheet of paper. It is important to see that the patient puts his weight on the foot during both parts of the process. The result is an accurate and permanent impression of the weight-bearing portions of the foot. 'The foot can be easily cleaned with soap and water.

FIG. 3.

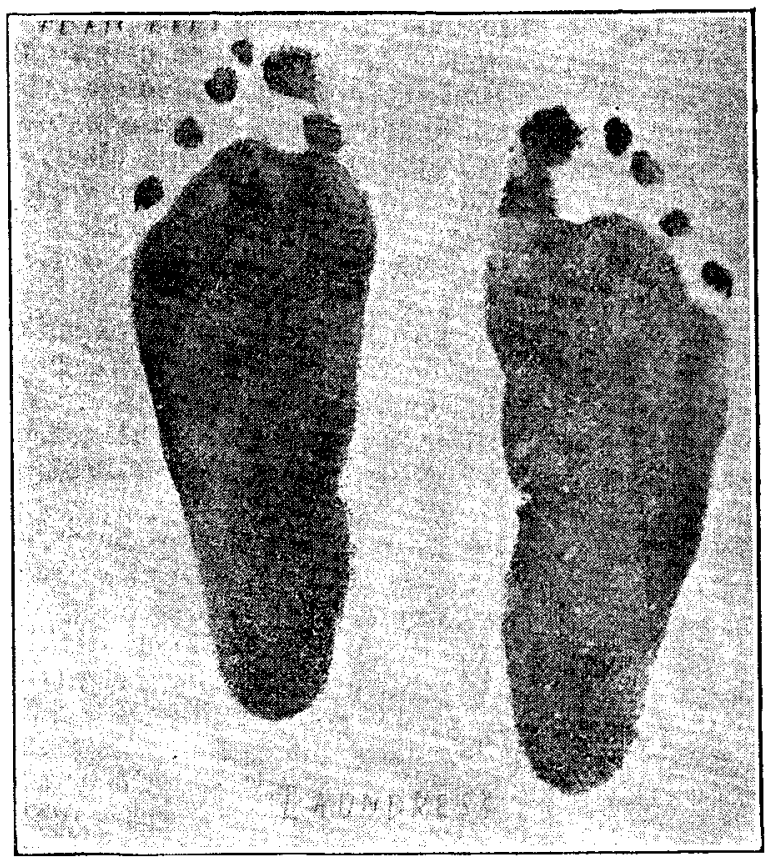

From a case of flat feet.

The impressions may be photographed and reduced in this way to a size suitable for the note-book. The illustration are from photographs of such impressions. A normal impression, Fig. 1, is by no means the rule. Fig. 2 shows the result of wearing bigh-heeled boots-viz., ces cavus with crowded toes. Fig. 3 is from a case of flat foot. The method is inexpensive and easy of application.

Llandudno.

\section{ORAL SEPSIS IN OPERATIONS ON THE THROAT.}

BY WYATT WINGRAVE, M.D. DURH.

PHYSICIAN TO THE CENTRAL IONDON THROAT AND EAR HOSPITAI.

So much attention has recently been paid to the connexion between oral sepsis and infective fevers that oral sepsis relative to operations upon the throat naturally presents itself for consideration. It seems almost superfluous to emphasise the necessity for careful cleansing of the parts before and after such operations, yet notwithstanding the elaborate precautions taken in other regions it must be admitted that in the more familiar cases of tonsillotomy, uvulotomy, \&c., the same amount of care is not always taken. This may be explained, in part, by the prevalence of the view that these cases generally "do well of their own accord "and that complications are extremely rare even in hospital out-patient practice; or that, unlike other regions, the mouth cannot be sterilised by ordinary methods, and if it were possible it could not be kept sterile for very long, its functions themselves preventing it. However great a measure of truth there may be in such contentions, they certainly do not justify a total disregard of ordinary cleanliness in the mouth, even if an ideal asepsis is unobtainable. That most of them do terminate satisfactorily in the absence of special precaution must be admitted. At the Central London Throat and Ear Hospital it is customary to see every case of tonsillotomy on the eighth day after operation, when quite 80 per cent. are either completely healed or nearly so. But there remain some patients in whom the "stump" is very unhealthy looking, disinclined to heal, and complicated with middle-ear infection and constitutional symptoms. Thes e are almost invariably the subjects of oral sepsis, mal nutrition, or neglected sanitary measures-personal and 
domestic. Further, they are mouth breathers. Mouth breathing is one of the chief causes of oral sepsis and being an obvious result of nasal obstruction it is practically impossible to maintain a healthy mouth unless nasal breathing be first restored. The bad habit, however, unfortunatelv too often persists for a long time after the cause is removed in spite of discipline and every effort to correct it.

Although insanitary surroundings of the patient may be credited with much responsibility for complications, it is remarkable how few children of even the poorest class, living in the filthiest surroundings, are ever subject to protracted healing or other complications when the simplest measures for insuring mouth cleanliness are carried out. It is chiefly in the debilitated and the neglected that they arise. Trouble from middle-ear infection in adenoid operations is now fortunately ve' $y$ rare among this class, a result not unreasonably attributed in no small degree to the prohibition of nasal douching. The healing of so large an area as th it occurring in a bilateral tonsillotomy, together with a "raw" naso-pharynx, must be influenced by a septic mouth. Ther $\Rightarrow$ need be therefore no doubt as to the expediency of adopting trustworthy corrective and preventive measures, not only before but during and after the operation. Bacteria may not only be reduced in number and virulence but the local conditions may be rendered as unfavourable as possible to their development.

The bacteria found in the mouth and the throat, both in apparently healthy and in diseased states, are so numerous that an exhaustive list would be too cumbrous in this article, but as it is customary arbitrarily to divide them into non-pathogenic and pathogenic it is necessary that in the present state of our knowledge a warning should be given of the danger of too strict a limitation. This specially applies to the moulds, since many of them are now recognised as pathogenic, and we have every reason to believe that under favourable conditions the list may be greatly extended by the inclusion of those which are at present classed non-pathogenic (1). ${ }^{1}$ For clinical evidence, bacteriological evidence, and published opinions are so conflicting that the pathogenicity, the specificity, or the harmlessness of most of them must not be too readily accepted or rejected, nor can too much caution be exercised in attributing to each one its respective power for good or ill. Both local and general powers of resistance and receptivity doubtless play important rôles, since it is not at all uncommon to find diphtheric and tubercle bacilli in the throats of healthy patients, likewise diplococci and strepto. cocci bacteriologically indistinguishable from the most virulent. To several bacteria have been dogmatically attributed individual clinical specificity-e g., the fusiform bacillus of Vincent, leptothrix, streptothrix, and spirochæta, yet each micro-organism may not only be found in the scrapings of almost any ulcer of the mouth and fauces but also in the apparently normal.

In six recent cases of persistent ulcers of the pharynx and tonsils of doubtful nature five of them afforded a pure growth of penicillium glaucum on serum and agar, while the sixth gave an equally pure crop of streptococcus pyogenes. In less than a week each case was healed and further cultures were negative. It must be admitted that at present clinical and bacteriological interpretation of bacteria occurring in this region are conflicting and most confusing. We must, therefore, not be too ready to accept as innocent or friendly a micro-organism which happens to be a "saprophyte," for in many of the moulds may be latent dangers almost as serious as those attending the more notorious pyogenic germ (2) (3)

Although the mouth, fauces, and oro-pharynx at all times may each be considered a perfect garden of germs it is fortunate thit under normal conditions the naso-pharynx is prat tically sterile. This may be due in part to the effective filtering action of the nostrils, the activity of the ciliated epithelium, but also in no small degree to the rich outflow of leucocytes and alexines from the healthy mucous membrane and lymphoid tissue of the parts. For whenever those tissues are diseased the cavity loses its sterility and swarms with bacteria. This is strikingly illustrated by Atrophic Rhinitis a disease in which the ciliated epithelium is replaced by stratified squamous cells and all the lymphoid elements entirely disappear, when the naso-pharynx is found to be swarming with micro-organisms and is offensively fotid. If we are right in attributing a protective power to these

1 The figures within parentheses refer to corresponding numbers in the bibliography at the end of the article. lymphoid structures surely special care is demanded after their wholesale suppression, as happens in tonsillotomy and the removal of adenoids.

How can the parts be cleansed? As already men. tioned, the naso-pharynx is normally sterile, but not always so in adenoids since they are not infrequently the seat of morbid changes, drainage and ventilation being more or less impaired. As a rule, however, the less an infant's nose is interfered with the better. Anterior or posterior nasal douching is not to be recommended (except in very rare cases) owing to the danger of infecting the middle-ear through the Eustachian tube. The careful introduction of boricated vaseline on a camel-hair brush, or the tip of the little finger, well into each nostril will excite sneezing and a copious nasal flow which generally clears the passage from accumulatinn. After the operation, in about from 12 to 24 hours, a solution of 1 per cent. of Peruvian balsam in liquid vaseline blown with an atomiser into the nostrils will keep the parts clean and sweet. If old enough, the child should at once be "disciplined" in gently blowing the nose, but violent efforts must not be allowe : until healing is complete and then only with great cuution. The nasopharynx will, as a rule, be kept quite clean by these measures apart from its own army of scavengers, the leucocytes. It need scarcely be added that under diseased conditions of the nose and naso-pharynx, such as an acute catarrh or atrophic rhinitis, all oral and throat operations should be undertaken with the strictest prec xution, if at all.

Cleansing of the mouth and also the fances is, however, far different and demands more energetic measures. As already stated, complete asepsis of this region is practically impossible but much can be done towards cleansing and keeping it clean. If the teeth are decayed a thorough treatment at the dentist's hands is indispensable, for no case should be operated upon at any age when suffering with carious teeth. The younger the patient the greater will be the difficulty, for infants and young children naturally resent all attempts to cleanse the mouth or throat, whatever method may be emplo ed. But, fortunately, the younger the child the lower is the septicity of the mouth (4), but with greater range in food, with increased mouth breathing and dental development, the greater is the likelihood of bacterinl development. Garsles are out of the question; therefore swabbing, irrigation, or direct application by sucking the different drugs must be resorted to in infancy and early childbood. At later ages, the patient's intelligence being relied upon, a wider range of selection will be arailable.

We will first deal with infants. Should there be any thrush, a gentle but thorough swabbing with glycerine of borax on a firmly applied cotton-wool mop every few hours and careful avoidance of re-infection will soon clear off the patches. B it strict assurance must be taken that the parts are quite free before attempting operation. Although there are many other far more rapid and thorough antiseptics, they are too irritating, too unpleasant in taste, or so harmful if swallowed as to impair their practicability. Boric acid in 10 per cent. solution, sanitas (1 per cent.), and lysoform ( $\frac{1}{2}$ per cent.) are all useful, especially the last, but for general use by the nurse a tablet of formamint, crushed and carefully inclosed in a piece of butter-cloth, the ends being secured by stout thread and held, may after moistening in water be placed in the infant's mouth for a few minutes several times in the course of the day. It readily dissolves in the saliva, has a pleasant taste, is non-poisonous, and yet most effectively controls bacterial growth and consequent fermentative processes. This is equally useful and trustworthy during the healing process, and is a method preferable to simply placing the tablet in the mouth, a somewhat dangerous practice at such an age (5).

Formamint, which has received much attention on the Continent and has already been referred to in this country is a loose chemical combination of formic aldehyde and lactose, put up in the form of a compressed tablet, each one containing one-sixth of a grain of formic aldehyde, combined with sugar of milk, citric acid, and pepto-hydrochloric acid, and flavouring agents. It differs entirely from a simple solution of formic aldehyde in nature and in action, being much more powerful, devoid of any irritating property, and on solution by the saliva sets free that disinfectant in a nascent form. It is maintained by $\mathrm{Z}$ willinger $(6)$ that it acts not only as a powerful local antiseptic but that it also continues its action after absorption as an antitoxin, so $a^{i}$ ding the reputed neutralising effect of healthy saliva upon 
toxins of oral origin as well as helping the immunising rôle of phagocytes in the pharynx.

In the case of older children and adults even more active measures are demanded, since a septic state of the mouth is generally either more pronounced or more likely to occur. In them even greater precautions with regard to the teeth must be taken, especially in cases involving treatment of the accessory nasal sinuses. A stronger mouth douche at such ages can be employed successfully, owing to the patient's intelligent coöperation, supplemented by the tooth-brush. Gargles are best avoided, especially when the parts are inflamed, from the great risk of swallowing the fluid or from causing pain and irritation due to its getting into the sensitive naso-ph rrynx and larynx. Few patients ever learn to gargle properly and failure only causes disappointment. Further, the act of gargling violently agitates structures which demand rest and passive treatment and there is the further risk, as in anterior and posterior nasal douching of infecting the middle ear when improperly performed. Many subjects of adenoids and enlarged tonsils are quite incapable of gargling owing to paresis of the soft pulate and faucial sphincter. In acute angina its painfulness is alone sufficient to indicate its inappropriateness when odynphagia is such a prominent symptom.

The most satisfactory substitute for gargle is either a mouth douche or a soluble lozenge. In case of the former the month is partially filled with solution, the head is tbrown back, and the lips are closed while the tongue's movements distribute the fluid. But by far the more thorough method is that of the lozenge as exempl fied in the formamint tablet This should be placed in the mouth and allowed to undergo passive solution rather than active mastication, a process which facilitates a closer contact and a wider di-tribution of the antiseptic, with but little discomfort and no danger to the patient.

The list of available mouth antiseptics is a very large one, but few of them are free from shortcomings however powerful their antiseptic action may be. First we have phenol, which, if employed in sufficient strength to kill bacteria, is not only poisonous but also unpleasant and temporarily destroys the sense of taste (and smell too if applied to the olfactory mucous membrane). Liquor carbonis detergens, creolin, cyllin, \&co, are equally unpleasant but less toxic and perhaps relatively stronger as bactericides, ret good deodorants. The different permanganates are certainly better, especially permanganate of zinc. This is not only ffectual but pleasant and far superior to the sodium and potassinm salts. One tablet (one-tenth of a grain) should be dissolved in half a tumblerful of water. Preparations of menthol, eucalyptol, thymol, and other volatile oils are only deodorants. Biborate and bicarbonate of sodium are useful adjuvants since they dissolve mucin, but, like boric acid, bave but slight antiseptic power, as also salicplic acid and its salts, which, further, are solvents of epithelium. Chlorinated soda is undoubtedly valuable, especially when there are specific alcers and much fœtor. Weak solutions of sulphurons acid readily kill "mould" deposits such as thrush, aspergillus forms, leptothrix, and spirochætæ. Sanitas and different preparations of peroxide of hydrogen are not only good disinfectants and deodorants, but they are especially valuable in being non-poisonous. Formalin must be used in very dilute solutions and only employed in the mouth owing to its pungency and irritative action upon the more delicate respiratory tract. Lysoform, however, is less pungent, and as a mouth douche in $\frac{1}{2}$ per cent. solutions it affords a highly antiseptic and powerful deodorant. Martindale's combination with lavender is pleasant and effective. It need scarcely be emphasised that the salts of mercury should only be used by the surgeon. A yetozone is not only useful in the mouth but may also be employed as a nasal spray (10 grains to one pint of water) in the same way as Löffler s solution. These antiseptics may be used not only in solutions of appropriate strength as mouth.washes or douches by the patients themselves, supplemented by a sterile tooth-brush, but also applied as stronger solutions by the surgeon only, on swabs or mops to the selected areas.

Nasal douches are sometimes necessary but should only be prescribed with great caution. The most efficient and least irritating is a weals solution of borax and sodium sulphate (1 per cent ) preceded by an insufflation of boric acid and formamint, one tablet being finely powdered and mixed with 100 grains of specially fine powder of boric acid or, when greater stimulation is required, $\frac{1}{2}$ per cent. of lysoform in boric acid, A strong antiseptic is not necessary, since the requisite cleanliness is certain to follow even slight stimulation by the outflow of exudate and leucocytes, which, together with the ciliary movement, can be relied upon effectively to cleanse the parts.

Although the foregoing selection includes but a few of many antiseptics, it is sufficiently representative to afford a tru-tworthy choice. Personal experience, however, enables the following preparations to be specially recommended: Formamint as a non-toxic and trustworthy antiseptic in all ages and all kinds of oral sepsis (it should be allowed passively to melt in the mouth, freely mixing with the saliva, and not masticated); permanganate of zinc for daily use and as a night and morning douche; lysoform, with or without lavender; sulphurous acid and liquor sodæ chlorinatæ in cases requiring a special preliminary swabbing and douching; and salicylic acid dissolved in borax when there is much epithelial accumulation.

But, as already mentioned, it is impossible to sterilise the mouth and keep it sterile by these or any other antiseptics. Nevertheless, the risk of auto.infection during operations in its neighbourhood may be greatly reduced by their intelligent employment.

3ibliography.-(1) Aspergillus Fumigatus. Baccarain : Gazzetta degli Osperiali, A pril 20th, 1906. Aerobic Moulds in Granulomata. LazarusBarlow: Transactions of the Pathological Society of London, 1903. Thrush. T. D. Lister: Transactions of the Patbological Society of London, 1898. (2) Steptothrix. Hahershon and Hichens: Transactions of the Pathological Society of London, 1901. Streptothrix. Emery Transactions of the Pathological Society of London, 19!3. (3) Pathogenic Mould-Snorothrix. Foulerton: Transactions of the Pathological Suciety of London, 1901. (4) Sterilıty of Month at Birth. Campo: Brit. Med, Jour Enitome, October, 1693. (5) Formaldehyde in Infectious Diseacec. ThF Lurcer, Dec $23 \mathrm{rd} 1905$ ) 1871 (quoting Schartion Stratford-place, $W$.

\section{$\mathbb{C l i n i t a l}$ glotes:}

MEDICAL, SURGICAL, OBSTETRICAL, AND THERAPEUTICAL.

\section{A CASE OF GRAVES'S DISEASE TREATED BY} RODAGEN.

By J. Royaldson Russell, M.R.C.S. ENG., L.R C.P. Lond.

RODAGEN is described as a mixture of milk-sugar and the desiccated milk of a goat from which the thyroid gland has been removed. It has been used with apparent success in cases of Graves's disease and the following is an account of a case in which I was able to try the effect of the drug. Messrs. R. W. Greeff, Eastcheap, London, kindly provided me with a supply of rodagen sufficient for a six weeks' trial. The previous history of the case before commencing rodagen was as follows. The patient was an unmarried woman aged 29 years. She first consulted me in May, 1905, on account of loss of weight amounting to 14 pounds in three months. She suffered from profuse perspirations; exophthalmos was marked, and neither Stellwag's nor von Graefe's signs were present. There was uniform slight enlargement of the thyroid. Tachycardia was presert, the pulse varying from 120 to 160 per minute. No cardiac murmur could be detected but the pulsations of the thyroid and of the large vessels of the neck were noticeable to the patient herself. Tremor of the hands was present and a feeling of weakness in the knees. She was sent to a farmhouse, where she had four pints of milk per diem in addition to ordinary diet, and was kept at rest in the recumbent position out of-doors for one month. She was also given bromide and tincture of digitalis but without apparent benefit from these. On the other hand, the dieting and the rest apparently were of large benefit to her and all the symptoms became less severe and she increased in weight 7 pounds. Treatment by rodagen was commenced on June $17 \mathrm{th}, 1906$, her weight being then 9 stones 4 pounds. She was first given five grammes per diem and afterwards 10 grammes. On July 16 th her weight had increased to 9 stones 7 pounds and on August 7 th she weighed 9 stones 12 pounds. At this date the treatment was dircontinued. The weather during the whole of the time she was taking rodagen was very hot and the patient said that she was never so well in hot weather. Since taking the rodagen she states 